\title{
Dielectrophoretic response of DNA shows different conduction mechanisms for poly(dG)-poly(dC) and poly(dA)-poly(dT) in solution
}

\begin{abstract}
A.S. Mohamad 1,2 , J. C. Jeynes 3 , M.P. Hughes ${ }^{1^{*}}$
${ }^{1}$ Centre for Biomedical Engineering, Department of Mechanical Engineering Sciences, University of Surrey, Guildford, GU2 7XH, UK

${ }^{2}$ Medical Engineering Technology Department, Universiti Kuala Lumpur, Selangor, 53100, Malaysia

${ }^{3}$ Advanced Technology Institute, University of Surrey, Guildford, GU2 7XH, UK.

*Corresponding author. e-mail: m.hughes@surrey.ac.uk., telephone +44 1483 686775

Abstract

In this paper we use dielectrophoresis to determine the electrical properties of poly(dG)-poly(dC) (GC) and poly(dA)-poly(dT) (AT) DNA in solution. The molecules show different conduction mechanisms, with GC DNA predominately through the molecule, but that conduction through the counterion cloud surrounding the molecule in solution is the more significant conductor in AT DNA. Moreover, the effect of frequency on DNA lengths differing by four orders of magnitude show no difference in the dielectrophoretic response.
\end{abstract}




\section{Introduction}

Dielectrophoresis is a phenomenon which has demonstrated increasing potential for molecular characterisation over the last 10-15 years. Dielectrophoresis occurs when an AC field is applied to particles in solution, inducing a dipole on the particle and interacting with that dipole to induce a force towards areas of high or low field intensities [1]. For any given molecule, a relaxation time $(\tau)$ can usually be calculated by the frequency response of the induced dipole which depends on the speed at which charge carriers can rearrange themselves in response to an electric field. The first studies of DNA dielectrophoresis were carried out by Washizu et al who found that DNA could be manipulated and stretched at high electric field intensities between frequencies of $40 \mathrm{kHz}$ and $2 \mathrm{MHz}$ [2]. Despite some studies into the underlying mechanism on a molecular level for DNA dielectrophoresis, a fully comprehensive understanding has not yet been provided.

A few groups have attempted to categorise the behaviour of DNA during dielectrophoresis in terms of electric field strength, frequency, length, single stranded or double stranded, ion concentration, viscoscity and pH. Asbury et al found that DNA trapping between microfabricated metal electrodes became more efficient as the frequency was lowered from $2 \mathrm{kHz}$ down to $10 \mathrm{~Hz}$ but that there was no significant difference between the trapping of different sized DNA molecules (ranging from $4 \mathrm{Kbp}$ to $164 \mathrm{Kbps}$ ) and the process was governed by a single relaxation time [3]. In contrast however, Chou et al showed that smaller molecules of DNA took less force to be attracted to the electrodes than larger ones [4]. However, they used a different experimental setup preferring an electrodeless array to prevent electrolysis at low frequencies rather than using metal electrodes. Electrolysis and electrothermal currents have been observed in other DNA experiments. Germishuizen et al found in their study of the factors affecting elongation of DNA, that DNA was pushed away from the centre of electrodes by fluid flow at frequencies below $100 \mathrm{kHz}$ [5]. Bakewell et al investigated the time dependant collection of DNA in a high frequency range $100 \mathrm{kHz}$ to $5 \mathrm{MHz}$ [6] to avoid electrolysis and electrothermal currents and found less collection at higher frequencies. Moreover, they found similar trends in the frequency dependant real part of polarisability using dielectrophoretic spectroscopy[7].

Both Chou et al. [4] and Asbury et al. [3] also investigated the hypothesis that the counterion cloud surrounding the backbone of the DNA was responsible for the induced dipole, rather than charge being carried through the DNA molecule. Chou et al found a clear shift of the frequency of the maximum force response to lower frequencies with increasing viscosities, indicating that the charge was carried on the counterion cloud [4]. Asbury et al varied the ionic composition of the solution and found a significant decrease in trapping efficiency above $1 \mathrm{mM}$ Tris, again indicating that the dipole moment is effected by the counterions [3]

The conductive properties of DNA have been hotly debated, with authors proposing that it is semiconducting [9] or metallic [10] although it seems that a 
general consensus that double stranded (ds) DNA on untreated $\mathrm{SiO}_{2}$ or mica is insulating $[11,12]$. One report claims that AT acts like a n-type semiconductor while GC acts like an p-type semiconductor [13]. However, all of the many different techniques which have been used to probe the conducting properties of DNA have considerable drawbacks. Those conducted on dehydrated DNA molecules do not take into account the fact that direct electronic conductivity, by means of overlapping $\pi$-orbitals of the base pairs along the molecular axis, is likely to be sensitive to helical conformations in (ds)DNA. The deformations in structure can be attributed to, for example, the humidity or the interaction between the molecule and a substrate [14]. Indeed, it has been shown that (ds)DNA on graphite in moist conditions appears in its natural B-form whereas it collapses to the A form (overlapping $\pi$-orbitals) in dry conditions [15]. Moreover, a recent report shows that the conductivity increases between DNA trapped between electrodes from a few $\mathrm{T} \Omega$ in dry conditions to a few $\mathrm{G} \Omega$ in moist conditions, even decreasing to a few $\mathrm{M} \Omega$ after many hours in a moist environment [16]. Hole injection studies carried out in solution have found that of the four DNA bases, guanine is the most easily oxidised, leading to a guanine radical cation $G^{\bullet+}[17,18]$ which is able to oxidise $G_{2}$. The newly formed guanine radical cation $G_{2}$ can then oxidise another guanine $\left(G_{3}\right.$, etc) so that the positive charge is transported by several tunnelling steps through DNA $[19,20]$. But these experiments cannot be carried out on very long lengths of DNA. Work by Xu et al [8] using short lengths of DNA was able to demonstrate differences in conduction in GC DNA into which AT bases were inserted, and suggested that conduction occurs through the molecule (as opposed to through the ion cloud).

In this paper, we investigate whether the AT or GC content of the DNA has any effect on dielectrophoresis. The frequency response of AT and GC DNA provides an indirect method of probing the conductivity of the molecules. Our experiments on GC and AT DNA using dielectrophoresis bypass these problems as the DNA is in its natural hydrated form, in long lengths and the effects of counterions and water are taken into account. If the dipole moment relies solely on the counterion cloud that surrounds the molecules in solution, then the frequency response should be the same between the AT and GC DNA. On the other hand, if the molecules themselves conduct differently and influence the dipole moment, then a difference should be observed in the frequency response.

\section{Materials and Methods}

Poly AT and poly GC DNA were purchased from Sigma Aldrich UK with length of $9 \mathrm{kbp}$ and $10 \mathrm{kbp}$. The concentration of poly AT and poly GC DNA of doublestranded homopolymer were prepared. The sample of $1 \mathrm{M} \mathrm{KCl}$ solution $(3.72 \mathrm{~g}$ $\mathrm{KCl}$ added to $50 \mathrm{ml}$ of Milli-Q water system) was used. DNA was stained with DAPI and J0JO-1 iodide (Sigma Aldrich, UK) to investigate any potential effects due to dye (none were observed).

Electrodes constructed of $100 \mathrm{~nm}$ gold film on a $10 \mathrm{~nm}$ titanium seed layer on glass were patterned and pholithographically etched with a quadrupolar design with $2 \mu \mathrm{m}$ gap between adjacent electrodes and $6 \mu \mathrm{m}$ between opposing 
electrodes [21]. AC potentials with magnitudes of between 5-10 Vp-p and frequencies from $100 \mathrm{kHz}$ to $10 \mathrm{MHz}$ were applied across the electrodes using a function generator (Thurby Thandar Instruments, TG120, UK). A fluorescence microscope (Eclipse E-400, Nikon Photonic Science, Japan) was used to observe the DEP behaviour of DNA molecules and the performances of the DEP trapping platforms. Data were collected using the crossover method whereby the frequency at which DEP collection ceases was observed for medium conductivities between $1 \mathrm{mS} / \mathrm{m}$ and $1 \mathrm{~S} / \mathrm{m}$; starting at a low voltage, the frequency was increased until collection ceased, the voltage was then raised and the procedure repeated.

\section{Results and Discussion}

The crossover frequency responses for both AT and GC DNA between $1 \mathrm{mS} / \mathrm{m}$ and $1 \mathrm{~S} / \mathrm{m}$ is shown in figure 1 , together with a best-fit theoretical model. Previous work [22] on the use of the crossover method for the analysis of nanoparticles has indicated that the behaviour of homogeneous ellipsoidal nanoparticles can be modelled successfully using

$$
F_{\text {rod }}=\frac{2 \pi r_{1} r_{2}^{2} \varepsilon_{m}}{3} \operatorname{Re}\left[f_{c m}\right] \nabla E^{2}
$$

Where $r_{1}$ and $r_{2}$ are the long and short radii of the ellipsoid, $\varepsilon_{m}$ is the medium permittivity, $\nabla$ is the gradient operator and $E$ is the magnitude of the electric field. $R e\left[f_{C M}\right]$ refers to the real part of the Clausius-Mossotti factor, which for an ellipsoid is given by the expression

$f_{C M}=\left[\frac{\varepsilon_{p}^{*}-\varepsilon_{m}^{*}}{\varepsilon_{m}^{*}+A\left(\varepsilon_{p}^{*}-\varepsilon_{m}^{*}\right)}\right]$

where $\varepsilon^{*}=\varepsilon-j \sigma, \varepsilon$ is permittivity, $\sigma$ is conductivity, $j=\sqrt{-1}$ and $\omega$ angular frequency, the subscripts $m$ and $p$ refer to the particle and medium respectively, and $A$ is a shape factor that tends to zero for high aspect ratios. Furthermore, the conductivity of the particle consists of multiple components corresponding to the effects of charge conduction through the particle and around the particle in the Stern and Diffuse parts of the electrical double layer

$\sigma_{p}=\sigma_{p b u l k}+\sigma_{s t e r n}+\sigma_{\text {diffuse }}$

where both of the first terms are indifferent to medium conductivity, making it difficult to discriminate between these two components by modelling. Therefore, for this study we will treat the particle conductivity as a combination of conduction through the molecule and that through charges bound to the molecule, but distinct from those in the diffuse ion cloud. 
The diffuse layer term differs in that the thickness of the diffuse double layer is dependent on the conductivity of the medium, and so observation of the behaviour with medium conductivity allows the determination of the $\zeta$-potential [23];

$$
\begin{aligned}
& K_{s}^{d}=\frac{\left(4 F^{2} c z^{2} D^{d}\left(1+3 m / z^{2}\right)\right)}{R T \kappa}\left(\cosh \left[\frac{z q \zeta}{2 k T}\right]-1\right)=\sigma^{d} \mu^{d} \\
& m=\left(\frac{R T}{F}\right)^{2} \frac{2 \varepsilon_{m}}{3 \eta D^{d}}
\end{aligned}
$$

where $D$ is the ion diffusion coefficient, $z$ the valence of the counterion, $F$ the Faraday constant, $R$ the gas constant and $T$ the temperature. $\sigma^{d}$ is the diffuse ion counter charge due to excess ions outside the slip plane and $\mu^{d}$ is the mobilitiy of these ions in the diffuse part of the double layer. $\kappa$ is the inverse Debye length, given by $\kappa=\sqrt{\left(\frac{2 c z F^{2}}{\varepsilon R T}\right)}$ with $c$ the electrolyte concentration $\left(\mathrm{mol} \mathrm{m}^{-3}\right)$, and $\eta$ is the viscosity. For a spherical particle of radius $r$, the diffuse layer conductivity is calculated thus:

$$
\sigma_{\text {diffuse }}=\frac{2 K_{s}^{d}}{r}
$$

However, although this term is more complex for ellipsoidal particles, where those particles are of similar dimensions, the expression allows comparison of relative values of $\zeta$-potential.

Finally, an additional component in the DEP behaviour was found at higher conductivities, for which we have added an additional relaxation with relaxation time $\tau_{e}$, which may represent the orientation dispersion of the molecule [24]. The best-fit models shown in figure 1 were constructed by modelling the real part of equation 2-5. For expressions 5, where ellipsoidal models are unavailable, a nominal value of $2 \mathrm{~nm}$ was used in order to determine a relative value for AT and GC DNA. The best fit was found using the parameter set shown in Table 1.

A number of interesting factors can be elucidated. The first is that the nondiffuse conduction (conduction principally through the molecule) is significantly larger for GC than AT, by over an order of magnitude. Other studies have suggested a difference in the conduction properties of AT and GC DNA; for example, work by Yoo et al [13] indicated a 70-fold increase in the conduction of dry GC DNA compared to AT, and suggested that the primary difference between dried AT and GC DNA conduction is down to different charge transport between the two base pair configurations. Whilst the difference here is of a smaller magnitude, the difference in conditions (wet vs. dry) may be a contributory factor. Similar effects were observed in aqueous solutions on GC DNA probed by AFM [8], where conduction was found to be proportional to the amount of AT 
inserted into a short strand of GC DNA. Since that DNA was primarily GC in origin, this concurs with our assertion that GC DNA is primarily electronic in conduction.

However, the second notable result is that the effective conduction in the diffuse layer is significantly higher in AT (by a factor 7), meaning that over the range of conductivities analysed the diffuse-layer conduction in AT is always larger than conduction through the molecule, whereas the converse is true in GC. The effect can be seen in the marked increase in crossover frequency with increasing conductivity in AT DNA, an effect strongly correlated with charge accumulation in the diffuse ion cloud, which reduces in size as the bulk medium conductivity increases and the Debye length reduces [23]. The origin for the increase in charge in the diffuse layer in AT DNA is as yet not established. Note that as indicated earlier, the values are relative due to the spherical approximation in equation 5, and as such the determined values only have value when used comparatively (as opposed to indicating absolute molecular stability).

Looking at the other parameters, the AT molecule appears to have a higher permittivity than GC, which may be a function of the greater charge stored in the diffuse layer. Conversely, the time constant for the low-frequency dispersion $\tau_{e}$ is shorter for GC (290ns) than AT (400ns) indicting the dipole moment is influenced by intrinsic conduction rather than ion cloud conduction. Conversely, this dispersion could be related to the dispersion of the double layer itself, as has been shown for nanoparticles such as latex beads [25].

Whilst Yoo et al. [13] attributed DNA conduction to charge movement through the molecule, others such as Chou et al [4] and Asbury et al [3] have suggested that it is the ion cloud that dominates the electrical properties of DNA in solution. Alternatively, Tomić et al. [26] used dielectric spectroscopy between $40 \mathrm{~Hz}-$ $110 \mathrm{MHz}$ in four different ionic solutions, and their studies indicated that the conduction mechanism was such that it was impossible to distinguish between conduction in the molecule from that in the counterion cloud. This study indicates that in fact, both mechanisms might be true, but the balance between molecular and ion-cloud condition mechanisms will depend on the ratio of AT and GC bases. Clearly, further work will be needed to understand how the charge transport is affected by the positioning and mixture of the bases within the molecule.

\section{Acknowledgements}

ASM was supported by the Malaysian Government under Grant Skim Tindikan MARA. 


\section{Reference}

[1] H.A.Pohl, "Dielectrophoresis: the behavior of neutral matter in non-uniform electric fields", Cambridge University Press, 1978.

[2] M.Washizu, O.Kurosawa, Ieee Transactions on Industry Applications 1990, 26, 1165.

[3] C.L.Asbury, A.H.Diercks, G.van den Engh, Electrophoresis 2002, 23, 2658.

[4] C.F.Chou, J.O.Tegenfeldt, O.Bakajin, S.S.Chan, E.C.Cox, N.Darnton, T.Duke, R.H.Austin, Biophysical Journal 2002, 83, 2170.

[5] W.A.Germishuizen, P.Tosch, A.P.J.Middelberg, C.Walti, A.G.Davies, R.Wirtz, M.Pepper, Journal of Applied Physics 2005, 97.

[6] D.J.Bakewell, H.Morgan, IEEE Transactions on Nanobioscience 2006, 5, 139.

[7] D.J.Bakewell, I.Ermolina, H.Morgan, J.Milner, Y.Feldman, Biochimica et Biophysica Acta-Gene Structure and Expression 2000, 1493, 151.

[8]B.Xu, P.Zhang, X. Li, N.Tao, Nano Letters 2004, 4, 1105.

[9] D.Porath, A.Bezryadin, S.de Vries, C.Dekker, Nature 2000, 403, 635.

[10] H.W.Fink, C.Schonenberger, Nature 1999, 398, 407.

[11] P.J.de Pablo, F.Moreno-Herrero, J.Colchero, J.G.Herrero, P.Herrero, A.M.Baro, P.Ordejon, J.M.Soler, E.Artacho, Physical Review Letters 2000, 85, 4992.

[12] A.J.Storm, J.van Noort, S.de Vries, C.Dekker, Applied Physics Letters 2001, 79, 3881.

[13] K.H.Yoo, D.H.Ha, J.O.Lee, J.W.Park, J.Kim, J.J.Kim, H.Y.Lee, T.Kawai, H.Y.Choi, Physical Review Letters 2001, 87, 198102.

[14] A.Y.Kasumov, D.V.Klinov, P.E.Roche, S.Gueron, H.Bouchiat, Applied Physics Letters 2004, 84, 1007.

[15] M.H.Zareie, P.B.Lukins, Biochemical and Biophysical Research Communications 2003, 303, 153.

[16] S.Tuukkanen, A.Kuzyk, J.J.Toppari, V.P.Hytonen, T.Ihalainen, P.Torma, Applied Physics Letters 2005, 87.

[17] C.A.M.Seidel, A.Schulz, M.H.M.Sauer, Journal of Physical Chemistry 1996, 100, 5541.

[18] S.Steenken, S.V.Jovanovic, Journal of the American Chemical Society 1997, $119,617$.

[19] J.Jortner, M.Bixon, T.Langenbacher, M.E.Michel-Beyerle, Proceedings of the National Academy of Sciences of the United States of America 1998, 95, 12759.

[20] E.Meggers, M.E.Michel-Beyerle, B.Giese, Journal of the American Chemical Society 1998, 120, 12950.

[21] N.G.Green, H.Morgan, J.J.Milner, Journal of Biochemical and Biophysical Methods 1997, 35, 89.

[22] N.G.Green, H.Morgan Journal of Electrostatics 1997, 42, 279.

[23] M.P.Hughes, H.Morgan, M.F. Flynn Journal of Colloid and Interface Science 1999, 220, 454.

[24] K.Yamaoka, K.Matsuda, Macromolecules 1980, 13, 1558.

[25] M.P.Hughes Journal of Colloid and Interface Science 2002, 250, 291.

[26] S.Tomić, S. Dolanski Babić, T.Vuletić, S.Krča, D.Ivanković, L.Griparić, R.Podgornik, Physical Review E 2006, 75, 021905 


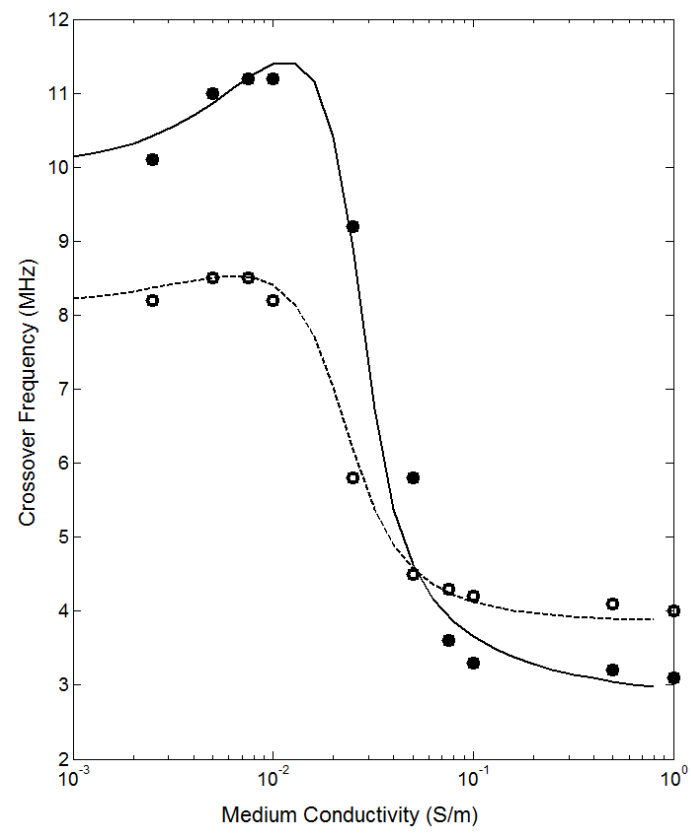

Figure 1. DEP crossover frequency vs. medium conductivity for AT (filled dots) and GC (open dots) DNA, with best-fit models (solid and broken lines, respectively).

Table 1. Parameters used to construct the best-fit models of Figure 1. Note that the zeta potential is a relative, rather than absolute, value.

\begin{tabular}{lll}
\hline & GC & AT \\
\hline$\sigma(\mathrm{mS} / \mathrm{m})$ & 10 & 0.9 \\
$\tau_{e}(\mu \mathrm{s})$ & 0.29 & 0.4 \\
Relative $\zeta(\mathrm{mV})$ & -2 & -14 \\
$\varepsilon_{p}$ & 60 & 72 \\
\hline
\end{tabular}

